\title{
A NOVEL TEXTURE DESCRIPTOR: CIRCULAR PARTS LOCAL BINARY PATTERN
}

\author{
IbTissam Al SAIDi ${ }^{1}$, MOHAMmed RZIZA ${ }^{1}$ AND JOHAN DEBAYLE ${ }^{\varpi, 2}$ \\ ${ }^{1}$ LRIT Laboratory, CNRST URAC 29, Rabat IT Center, Faculty of Sciences, Mohammed V University in Rabat, \\ B.P. 1014 Rabat, Morocco, ${ }^{2}$ Mines Saint-Etienne, CNRS, UMR 5307 LGF, Centre SPIN 158 cours Fauriel, \\ 42023 SAINT-ETIENNE Cedex 2, France \\ e-mail: ibtissam_alsaidi@um5.ac.ma,mohammed.rziza@gmail.com,debayle@emse.fr \\ (Received May 30, 2021; accepted June 26, 2021)
}

\begin{abstract}
Local Binary Pattern (LBP) are considered as a classical descriptor for texture analysis, it has mostly been used in pattern recognition and computer vision applications. However, the LBP gets information from a restricted number of local neighbors which is not enough to describe texture information, and the other descriptors that get a large number of local neighbors suffer from a large dimensionality and consume much time. In this regard, we propose a novel descriptor for texture classification known as Circular Parts Local Binary Pattern (CPLBP) which is designed to enhance LBP by extending the area of neighborhood from one to a region of neighbors using polar coordinates that permit to capture more discriminating relationships that exists amongst the pixels in the local neighborhood which increase efficiency in extracting features. Firstly, the circle is divided into regions with a specific radius and angle. After that, we calculate the average gray-level value of each part. Finally, the value of the center pixel is compared with these average values. The relevance of the proposed idea is validate in databases Outex 10 and 12. A complete evaluation on benchmark data sets reveals CPLBP's high performance. CPLBP generates the score of 99.95 with SVM classification.
\end{abstract}

Keywords: Feature extraction, Local binary pattern (LBP), Texture classification.

\section{INTRODUCTION}

Texture is a basic characteristic of the appearance of various natural areas. It is an essential component of computer vision systems, and is omnipresent in natural images. Texture classification, as one of the big issues in texture analysis, has been a longstanding research topic in the domain of pattern recognition and computer vision, because of its importance firstly for understanding how the process of texture recognition is employed in human vision and secondly for the significant role it plays in the large diversity of applications of image analysis [Pietikainen et al., 2011]. The texture classification is used in various applications (e.g.biomedical image analysis, face recognition, remote sensing, face analysis and biometrics, document classification, object recognition, fabric inspection) [Tuceryan and Jain, 1993]. The analysis of real world texture has shown to be surprisingly difficult caused by the natural texture inhomogeneity of scale and rotation changes, variability in surface shape and illumination.

Like the classical problem of pattern recognition, texture classification mainly comprises of two sub issues: feature extraction and classifier design [Pietikainen et al., 2011], [Tuceryan and Jain, 1993]. It is globally agreed that the most significant role is the extraction of strong texture features to get a good recognition result, because if poor features are employed even the best classifier will fail to attain great results caused by various imaging distortions (including illumination, rotation, view point, scaling, occlusion, nonrigid deformations and noise). Therefore, the research works in the literature are mostly concentrated on the feature extraction part in the texture classification. Furthermore, an excellent survey given in [Pietikainen et al., 2011, Brahnam et al., 2014] of various textures feature extraction methods have been developed.

For getting the best texture classification with images that captured beneath changing pose condition or illumination, we need to focus on the variations of grayscale, scale and rotation. Various approaches and models have been proposed to obtain gray scale and rotation invariant texture classification such as hidden Markov model [Chen and Kundu, 1994], autoregressive model [Kashyap and Khotanzad, 1986], Gaussian Markov model [Deng and Clausi, 2004]. Recently, [Cui et al., 2006] and [Liu et al., 2009] introduce a texture classification method, which employs a rotation-invariant feature derived from the radon transform [Deans , 2007]. Local binary pattern (LBP) approaches have also been utilized in the texture analysis domain with rotation and scale invariance [Guo et al., 2010a, Liu et al., 2015]. 
Among the various texture features extraction methods, Local Binary Pattern (LBP) [Ojala et al., 2002] have appeared as one of the most significant and broad-studied classes of texture features. Owing to their remarkable advantages (easy to implement and low computational complexity), LBP has evolved rapidly in the domain of computer vision and image analysis. Indeed, for a various range of issues several LBP variants have been proposed in the literature: face recognition [Ruiz-del Solar and Quinteros, 2008], dynamic texture recognition [Zhao and Pietikainen, 2007], texture classification [Ojala et al., 2002, Liao et al., 2009], face image analysis [Ahonen et al., 2006], biomedical image analysis [Nanni et al., 2010], object detection [Trefny and Matas, 2010, Nguyen et al., 2013].

[Ojala et al., 2002] introduced Local Binary Pattern (LBP) histogram for grayscale and rotation invariant texture classification. Although the original LBP method had important limits, the general philosophy of LBP has proven very popular owing to its flexibility and efficiency, furthermore the various modifications and extension were introduced to enhance their robustness and performance. [Liao et al., 2009] proposed Dominant Local Binary Pattern (DLBP) that eliminate seldom-occurred patterns by using the most frequency ones. [Bianconi et al., 2015] was investigated the problem of determining sets of discriminative patterns by answring a specific question which is the best labelled methods or unlabelled methods. [Guo et al., 2010a] was the first ones that introduced Completed Local Binary Pattern (CLBP) which combine various LBP features: CLBPS, CLBPM where the signs and magnitudes of local differences are done into two complementary components and CLBPC which is the same as the original LBP. LBPV [Guo et al., 2010b] was introduced to incorporate the globally rotation invariant matching with locally variant LBP texture features. After that DRLBP [Satpathy et al., 2014] was suggested to mix gradient magnitude information with LBP. Later, RLBP was introduced by [Chen et al., 2013] to be rugged to the noise existed in the image by modifying the coding of bits of LBP. Recently, [Liu et al., 2016] proposed MRELBP which regrouped MRELBP-NI, MRELBP-RD and MRELBPCI in order to upgrade the noise ruggedness and discriminative power furthermore to take both microtexture and macrotexture texture information. [Guo et al., 2016] suggested SSLBP, which combined SSLBPCS and SSLBPCM founded on DLBP [Liao et al., 2009] in scale space, specifically in scale variation for texture classification. [Tabatabaei and Chalechale, 2019] proposed directional thresholded LBP (DTLBP) for noise-tolerant image feature extraction using average of the directional neighboring pixels values for thresholding to reduce noise.

In texture analysis, we can notice that the majority of the LBP methods use a limited number of neighboring pixels, therefore local textural information is loot. With the aim to improve the performance of LBP in texture classification, an effective extension of LBP for feature extraction is proposed in this paper named Circular Parts Local Binary Pattern (CPLBP). The CPLBP substitutes each neighborhood with the region of pixel neighbors (circular parts) specified by an angle and radius which permit to keep all the spatial information, after that each center is thresholded with the average of each region. The important properties such as rotation and scale invariance are more robust than LBP and based on the impact of the polar coordinate (radius $\mathrm{R}$ and angle $\theta$ ). Furthermore, computing the mean of each part of the circle allows to capture more information than the neighboring pixels and help to reduce the noise. According to the experimental results, we demonstrate that the suggested LBP extension can enhance the performance in terms of the classification accuracy.

The paper is organized as follows: a brief review of the original LBP is presented after introduction, our approach CPLBP is presented in the second section. In Section 3 we show the experimental results, followed by discussion and conclusion in the last Section.

\section{MATERIALS AND METHODS}

\section{LOCAL BINARY PATTERN (LBP)}

The original LBP [Ojala et al., 2002] is considered as a texture analysis for grayscale images, which characterizes the local spatial patterns of texture image patch through comparing the center pixel with his neighbors to make a string of binary bits that will be encoded to a decimal number. The way to calculate LBP is as follows:

$$
L B P_{r, N}(c)=\sum_{i=0}^{N-1} s\left(g_{i}-g_{c}\right) 2^{i}, s(x)= \begin{cases}1 & x \geq 0 \\ 0 & x<0\end{cases}
$$

where $N$ is the number of pixels in the neighbors, $r$ is the radius of neighbors, $g_{c}$ is the grey level of the central pixel of the neighbourhood and $s()$ is the sign function. If we assume that the coordinates of center pixel $g_{c}$ is $(0,0)$, then the coordinates of the neighbors are calculated as follows : $(-r \sin (2 \pi n / N), r \cos (2 \pi n / N))$. Furthermore, on sampling circle, the majority of sampling points do not correspond to the right pixel position specially 
for circular sampling pattern, a bilinear interpolation is generally required. Fig. 1 shows the circularly symmetric neighbor sets of LBP.

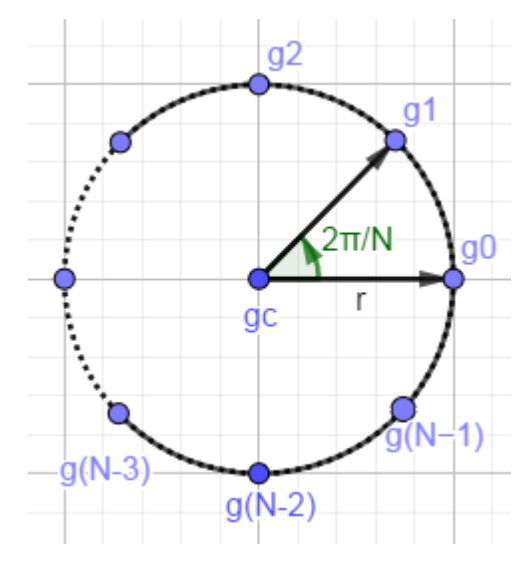

Fig. 1. The neighbor points used in the LBP method.

[Ojala et al., 2002] introduce a rotation invariant version $L B P_{(r, N)}^{r i}$ of $L B P_{(r, N)}$. This version aims to group together the LBPs which are actually rotated versions of the same pattern. After that, they noted that certain LBP patterns often occur than others, so the uniform LBP are used to only conserve the uniform patterns and groups all the other patterns as a nonuniform. A pattern is considered as uniform, if the number of spatial transitions in the sequence between 0 and 1 is lower or more equal to two. For example, the model LBP 00000100 is uniform (transition twice) and 00001010 is not uniform (transition 4 times). In the same paper Ojala proposed the rotation invariant uniform LBP descriptor to get an improved rotation invariance as well as to minimize the feature dimensionality, this proposition regroups two versions of $L B P_{(r, N)}\left(L B P_{(r, N)}^{r i}\right)$ and the uniform $L B P_{(r, N)}$ $\left(L B P_{(r, N)}^{u}\right)$. In particular, the rotation invariant uniform LBP is defined as:

$L B P_{r, N}^{r i u 2}=\left\{\begin{array}{rc}\sum_{i=0}^{N-1} s\left(g_{i}-g_{c}\right) 2^{i}, & \text { if } U\left(L B P_{r, N}\right) \geq 2 \\ p+1 & \text { otherwise }\end{array}\right.$

where the uniform LBP is given by:

$$
U\left(L B P_{r, N}\right)=\sum_{i=1}^{N}\left|s\left(g_{\bmod (i, N)}-g_{c}\right)-s\left(g_{i-1}-g_{c}\right)\right| .
$$

where the function $\bmod (i, N)$ is the modulus of $i$ to $N$

\section{THE PROPOSED METHODS}

This paper presents a new extension of LBP method for texture classification framework.

\section{CPLBP (CIRCULAR PARTS LOCAL BINARY PATTERN ) CLASSIFICATION METHOD}

Given the fact that the traditional encoding strategy of LBP uses a specific number of neighbors, even though the changing of radius that leads to loose the information. Our proposed method tries to keep all the information coming from neighbors by calculating the average of all pixels values from each part of the circle specified by angle and radius values (using polar coordinate system). Therefore, the CPLBP is discriminative and robust comparing to LBP due to the fact that it is designed to contain more spatial information.

Our approach named Circular Parts Local Binary Pattern (CPLBP) can be considered as an extension of the original LBP given by:

$C P L B P_{R, P}(c)=\sum_{p=0}^{P-1} s\left(g M_{p}-g_{c}\right) 2^{p}, s(x)= \begin{cases}1 & x \geq 0 \\ 0 & x<0\end{cases}$

Like LBP the central pixel $g_{c}$ intensity is the gray level of the central pixel of the neighborhood and $s()$ is the sign function. $R=r 1+r 2+r 3+\ldots+r n$ represent radius, $P$ is the circle part and $g M_{p}$ is the average pixels intensity of the circle part $P$. Since we are working in the sampling circle as LBP. If we suppose that $(0,0)$ are the coordinates of the center pixel $g_{c}$, then the coordinates of neighbors which are included in the circle part are calculated as follow: $(-R \sin (2 \pi n / P), R \cos (2 \pi n / P))$ where $R=r 1+r 2+$ $r 3+\ldots+r n$.

The following steps to get CPLBP are the following:

1. Divide the circle into parts. Choosing the radius $R$ and the angle $\theta$ of the circular part. These parameters $(R, \theta)$ enable to partition the circle of all pixel neighbors into parts. Each part is a region of pixel neighbors. The number of parts depends on the choice of the angle $\theta$. For example, if $\theta=$ 30 the circle will be divided into 12 parts.

2. Calculating the value of $g M_{p}$. Each part of the circle according to their radius and angle include a region of pixel neighbors, which are grouped to compute the average value of the circle part. 
3. Computing the Local Binary Pattern. In this step the value of the center pixel $g_{c}$ is compared with the average value of each part of the circle $g M_{p}$ to form the LBP encoded pattern.

In order to illustrate the proposed CPLBP. Fig. 2 shows the entire procedure with the example CPLBP $(8,3)$. As presented in Fig. 2, the important parameters of CPLBP are $\mathrm{R}$ and $\theta, R=r 1+r 2+r 3=3$ denotes the radius of the circle centered at pixel $g_{c}=161$, and we choose $\theta=45$ which allowed to divide the circle into 8 parts. Each part of pixel neighbors with $\theta=$ 45 exactly contains 6 neighbors . The average value of each six neighbors is calculated (in the example $" g M 1=160.33 ")$ and compared with the center $g_{c}=$ 161 to form the local binary code "01110100".

Note that the three properties Uniform, Rotation Invariant and Rotation Invariant Uniform are applicable for CPLBP.

\section{EXPERIMENTAL RESULTS}

\section{TEXTURE IMAGE DATASETS}

Our approach is tested using two of the publicly available texture databases known as Outex_TC_0010 and Outex_TC_0012. These databases are captured from a large variety of real material surfaces at nine different angles $(0.5,10,15,30,45,60,75$ and 90$)$, it includes 24 classes. Outex_TC_0010 has 180 texture images per class and each image has size $128 * 128$ under illumination 'Inca' while Outex_TC_0012 has 200 texture images per class and each image has size $128 * 128$ under several illumination conditions ('Inca', 'horizon', and 't184'). Some samples of this texture image database are shown in Fig. 3.

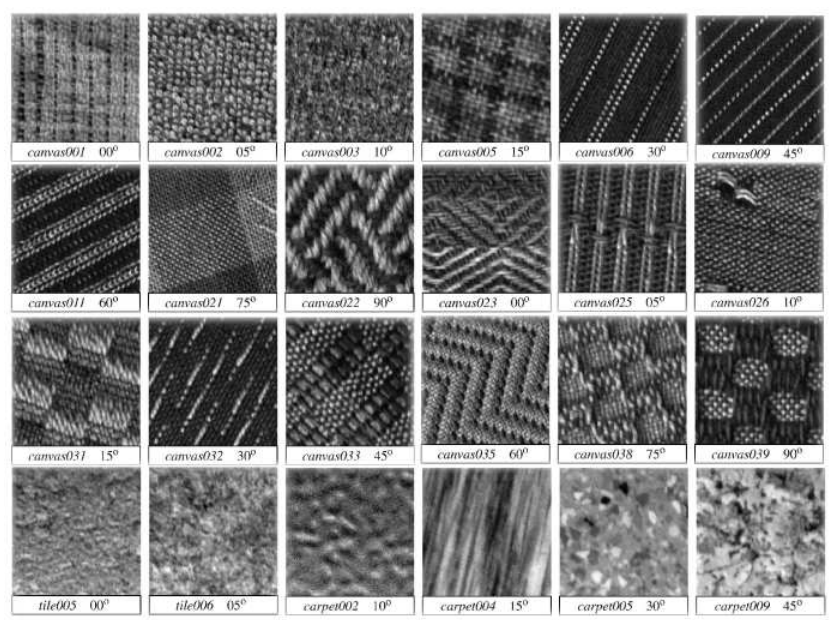

Fig. 3. Outex Texture Database.

\section{RESULTS}

To test the performance of the proposed approach, we use support vector machine (SVM) classifier method with the stratified 10 -fold cross validation to prevent over-fitting.

Considering this new proposed method CPLBP extensive experiments are used to demonstrate the performance of our method compared with other methods from the literature. In the experimental result 1 and 2 we will effectiveness observation CPLBP in the radius and angle change respectively.

\section{EXPERIMENTAL RESULTS 1}

This first experiment is done to evaluate the effect of thresholding the center pixel with the mean region of circular pixel neighbors. In this experiment, we compare CPLBP with LBP using both databases Outex_TC_0010 and Outex_TC_0012, employing different values of radius $(1,2,3,4,5,6,7,8,9$ and 10) and a fixed value of angle $\theta=45$ in order to reduce the feature dimension and running time.

Experiment 1-1: Radius evaluate CPLBP with LBP version

\section{On Outex_TC_0010}

Table 1 presents the comparative results of the classification accuracy of the proposed experiment CPLBP with different versions of LBP (classic, uniform (u2), rotation invariant uniform (riu2)) using Outex_TC_0010 . It can be noticed that our approach is enhanced in term of accuracies and achieved the best results for all versions: $\mathrm{CPLBP}=99.65, \mathrm{CPLBPu} 2$ $=99.05$ and CPLBPriu $2=98.24$ with the radius $\mathrm{R}=10$ contrary to LBP that lose their performance progressively: $\mathrm{LBP}$ classic $=96.11, \mathrm{LBPu} 2=90.07$ and LBPriu2 $=81.62$. This is mainly due to the impact of capturing much more discriminating information from local pixel neighbors existing in each part of circle specified by equal angle $\theta$ and represented with the average value, consequently they maintain their robustness especially when the radius increases.

\section{On Outex_TC_0012}

Table 2 illustrates the comparative results of CPLBP with LBP classic using two different illumination conditions Outex_TC_0012 "t184" and "horizon" with rotation changes. As can be seen from the results, our proposed approach provides also superior performance in term of average of all radius 94.61 and 95.20 Outex_TC_0012 ("horizon", and "t184") respectively, and mostly when the radius increase with $\mathrm{R}=1092.83$ for $\mathrm{T} 184$ and 92.75 for horizon. As mentioned before, this high performance is owing to: Firstly, on the impact of polar coordinate 

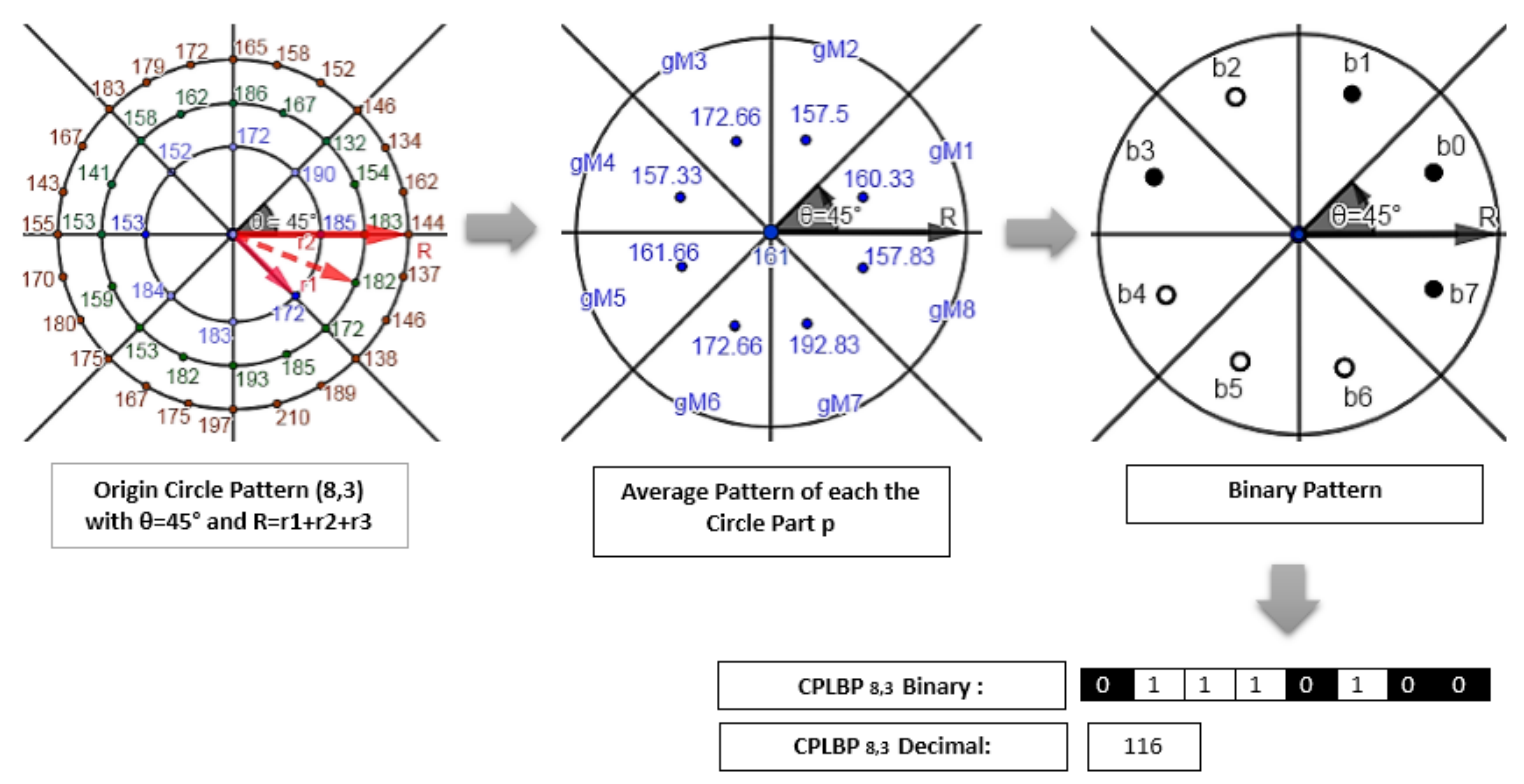

Fig. 2. The CPLBP of $(P=8, R=3)$.

Table 1. Classification accuracy (\%) of the CPLBP descriptor for different values of radius $R$ on the TC10 dataset.

\begin{tabular}{cccccccccccc} 
& \multicolumn{10}{c}{ Classification accuracy \% Outex_TC10 } \\
\cline { 2 - 11 } & $\mathrm{R}=1$ & $\mathrm{R}=2$ & $\mathrm{R}=3$ & $\mathrm{R}=4$ & $\mathrm{R}=5$ & $\mathrm{R}=6$ & $\mathrm{R}=7$ & $\mathrm{R}=8$ & $\mathrm{R}=9$ & $\mathrm{R}=10$ & Average \\
\hline LBP classic & 99.83 & 99.86 & 99.88 & 99.53 & 99.10 & 98.99 & 99.26 & 98.56 & 97.48 & 96.11 & 98.86 \\
CPLBP & $\mathbf{9 9 . 8 6}$ & $\mathbf{9 9 . 8 8}$ & $\mathbf{9 9 . 9 0}$ & $\mathbf{9 9 . 8 6}$ & $\mathbf{9 9 . 9 5}$ & $\mathbf{9 9 . 9 5}$ & $\mathbf{9 9 . 8 4}$ & $\mathbf{9 9 . 8 8}$ & $\mathbf{9 9 . 7 9}$ & $\mathbf{9 9 . 6 5}$ & $\mathbf{9 9 . 8 6}$ \\
\hline LBP u2 & 99.86 & $\mathbf{9 9 . 8 4}$ & 99.56 & 99.30 & 98.77 & 98.36 & 96.76 & 94.88 & 92.43 & 90.07 & 96.98 \\
CPLBP u2 & $\mathbf{9 9 . 8 8}$ & 99.67 & $\mathbf{9 9 . 7 7}$ & $\mathbf{9 9 . 7 9}$ & $\mathbf{9 9 . 7 0}$ & $\mathbf{9 9 . 7 2}$ & $\mathbf{9 9 . 7 5}$ & $\mathbf{9 9 . 6 3}$ & $\mathbf{9 9 . 5 1}$ & $\mathbf{9 9 . 0 5}$ & $\mathbf{9 9 . 6 5}$ \\
\hline LBP riu2 & $\mathbf{9 6 . 2 6}$ & $\mathbf{9 7 . 1 0}$ & 97.94 & 96.71 & 94.00 & 94.12 & 91.60 & 88.40 & 84.58 & 81.62 & 92.23 \\
CPLBP riu2 & 95.30 & 96.06 & $\mathbf{9 8 . 0 1}$ & $\mathbf{9 8 . 9 1}$ & $\mathbf{9 9 . 2 6}$ & $\mathbf{9 8 . 7 7}$ & $\mathbf{9 8 . 3 6}$ & $\mathbf{9 8 . 3 1}$ & $\mathbf{9 8 . 0 5}$ & $\mathbf{9 8 . 2 4}$ & $\mathbf{9 7 . 9 3}$ \\
\hline
\end{tabular}

Table 2. Classification accuracy (\%) of the CPLBP descriptor for different values of radius $R$ on the TC12 dataset.

\begin{tabular}{cccccccccccc} 
& \multicolumn{10}{c}{ Classification accuracy \% Outex_TC12 } \\
\cline { 2 - 11 } & $\mathrm{R}=1$ & $\mathrm{R}=2$ & $\mathrm{R}=3$ & $\mathrm{R}=4$ & $\mathrm{R}=5$ & $\mathrm{R}=6$ & $\mathrm{R}=7$ & $\mathrm{R}=8$ & $\mathrm{R}=9$ & $\mathrm{R}=10$ & Average \\
\cline { 2 - 11 } & \multicolumn{10}{c}{ Outex_TC12 $\mathrm{T} 184$} \\
\hline LBP classic & 94.08 & $\mathbf{9 5 . 4 4}$ & 94.73 & 94.23 & 94.15 & 93.42 & 91.50 & 90.88 & 88.79 & 86.54 & 92.38 \\
CPLBP & $\mathbf{9 5 . 3 3}$ & 95.43 & $\mathbf{9 5 . 2 5}$ & $\mathbf{9 5 . 5 4}$ & $\mathbf{9 5 . 0 8}$ & $\mathbf{9 4 . 9 0}$ & $\mathbf{9 4 . 9 4}$ & $\mathbf{9 3 . 5 2}$ & $\mathbf{9 3 . 2 9}$ & $\mathbf{9 2 . 8 3}$ & $\mathbf{9 4 . 6 1}$ \\
\hline & \multicolumn{10}{c}{ Outex_TC12 horizon } \\
\hline LBP classic & 94.84 & 95.48 & 95.75 & 94.90 & 94.25 & 93.42 & 92.29 & 91.63 & 90.02 & 87.48 & 93.01 \\
CPLBP & $\mathbf{9 7 . 3 0}$ & $\mathbf{9 6 . 9 0}$ & $\mathbf{9 6 . 3 8}$ & $\mathbf{9 6 . 2 5}$ & $\mathbf{9 5 . 4 0}$ & $\mathbf{9 4 . 9 2}$ & $\mathbf{9 4 . 5 6}$ & $\mathbf{9 3 . 8 8}$ & $\mathbf{9 3 . 6 5}$ & $\mathbf{9 2 . 7 5}$ & $\mathbf{9 5 . 2 0}$ \\
\hline
\end{tabular}

system that helps to get new extension robust than LBP in term of rotation changes. Secondly, on the local region pixel neighbors captured in precise $\theta$ that bring more spatial information than just one neighbor from LBP.

Fig. 4 draws the comparison accuracies of LBP classic and CPLBP of both databases outex_TC_0010 and 0012 with ten different radius from 1 to 10 . Our descriptor outperforms the classical LBP (even with high radius they keep a good accurate).

\section{SOTA}

Experiment 1-2: Radius comparative CPLBP with

In order to examine the robustness, performance and other advantage of our approach CPLBP in texture classification, we pick out five classification approaches based on the state of art literature LBP methods. They comprise LTP [Tan and Triggs, 2007], RLBP [Mehta and Egiazarian, 2013], DRLBP [Satpathy et al., 2014], CLBP [Bianconi et al., 2015] .

Table 3 shows the comparative results of the classification accuracy(\%) for outex_TC_0010 and 0012 (t184 and horizon). We have made this following observations from the results of the experiments.

In term of mean accuracy, RLBP and DRLBP are better then LBP. This is because RLBP and DRLBP shift results in a rotation invariance, as the weights depend on the neighborhood which make it more robust to detect the rotation variation. CPLBP_S and CPLBP_M get the sign and magnitude features respectively. The LTP is generally greater then LBP because it takes into account tree relations between center pixel and its neighbors 'greater than', 


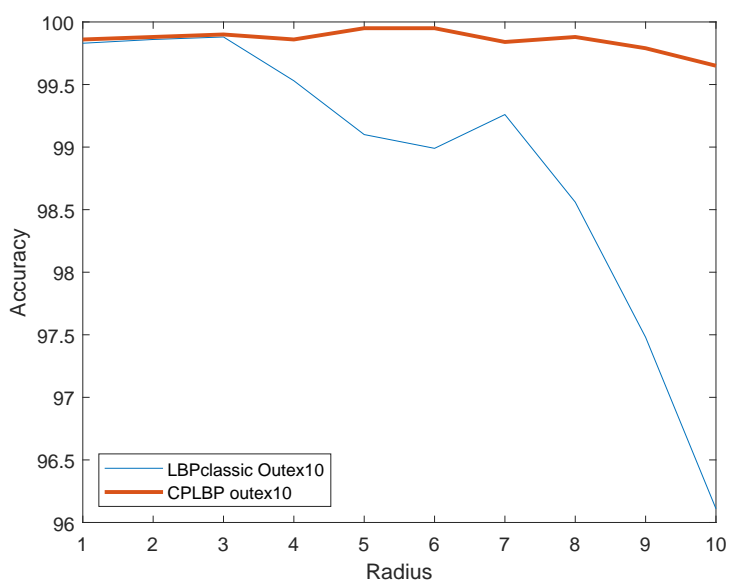

(a)

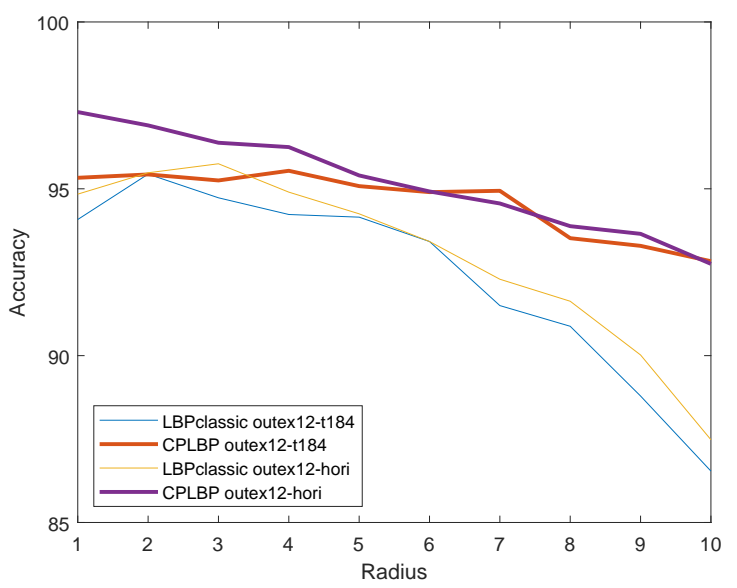

(b)

Fig. 4. The performance of CPLBP according to radius variation using (a) Outex_TC_0010 (b) Outex_TC_0012 t184 and horizon.

Table 3. Classification accuracy (\%) of the CPLBP descriptor with the state of the art methods for different values of $R$ on the outex_TC_0010n and 0012 databases.

\begin{tabular}{|c|c|c|c|c|c|c|c|c|c|c|c|}
\hline \multicolumn{12}{|c|}{ Classification accuracy \% Outex_TC 10 and 12} \\
\hline & $\mathrm{R}=1$ & $\mathrm{R}=2$ & $\mathrm{R}=3$ & $\mathrm{R}=4$ & $\mathrm{R}=5$ & $\mathrm{R}=6$ & $\mathrm{R}=7$ & $\mathrm{R}=8$ & $\mathrm{R}=9$ & $\mathrm{R}=10$ & Average \\
\hline & \multicolumn{11}{|c|}{ Outex_TC10 } \\
\hline LBP classic & 99.83 & 99.86 & 99.88 & 99.53 & 99.10 & 98.99 & 99.26 & 98.56 & 97.48 & 96.11 & 98.86 \\
\hline LTP & 99.84 & 99.93 & 99.95 & 99.91 & 99.88 & 99.88 & 99.68 & 99.00 & 98.77 & 97.92 & 99.48 \\
\hline RLBP & 99.35 & 99.81 & 99.84 & 99.95 & 99.86 & 99.93 & 99.51 & 98.40 & 98.63 & 97.69 & 99.40 \\
\hline DRLBP & 98.58 & 98.05 & 99.86 & 99.93 & 99.88 & 99.93 & 99.65 & 99.31 & 98.59 & 97.99 & 99.17 \\
\hline CLBP M & 98.94 & 99.58 & 99.81 & 99.72 & 99.75 & 99.49 & 99.03 & 98.13 & 97.82 & 96.94 & 98.92 \\
\hline CLBP S & 99.79 & 99.93 & 99.98 & 99.81 & 99.70 & 99.70 & 99.31 & 98.56 & 97.48 & 96.32 & 99.06 \\
\hline \multirow[t]{2}{*}{ CPLBP } & 99.86 & 99.88 & 99.90 & 99.86 & 99.95 & 99.95 & 99.84 & 99.88 & 99.79 & 99.65 & 99.86 \\
\hline & \multicolumn{11}{|c|}{ Outex_TC12 T184 } \\
\hline LBP classic & 94.08 & 95.44 & 94.73 & 94.23 & 94.15 & 93.42 & 91.50 & 90.88 & 88.79 & 86.54 & 92.38 \\
\hline LTP & 95.58 & 95.69 & 95.60 & 95.10 & 94.68 & 93.47 & 92.88 & 92.75 & 92.25 & 91.69 & 93.87 \\
\hline RLBP & 90.27 & 92.46 & 92.45 & 88.50 & 88.00 & 86.19 & 83.73 & 84.65 & 82.83 & 80.88 & 87.00 \\
\hline DRLBP & 90.11 & 90.81 & 94.41 & 90.19 & 89.00 & 87.15 & 85.10 & 85.47 & 83.44 & 82.80 & 87.85 \\
\hline CLBP M & 92.40 & 94.23 & 94.25 & 93.73 & 93.15 & 92.81 & 91.77 & 91.27 & 90.33 & 89.79 & 92.37 \\
\hline CLBP S & 96.52 & 96.35 & 96.29 & 96.10 & 95.47 & 94.50 & 93.60 & 92.13 & 91.73 & 90.00 & 94.27 \\
\hline CPLBP & 95.33 & 95.43 & 95.25 & 95.54 & 95.08 & 94.90 & 94.94 & 93.52 & 93.29 & 92.83 & 94.61 \\
\hline \multicolumn{12}{|c|}{ Outex_TC12 horizon } \\
\hline LBP classic & 94.84 & 95.48 & 95.75 & 94.90 & 94.25 & 93.42 & 92.29 & 91.63 & 90.02 & 87.48 & 93.01 \\
\hline LTP & 96.33 & 95.73 & 95.88 & 95.15 & 94.79 & 94.42 & 93.38 & 93.77 & 93.10 & 92.06 & 94.46 \\
\hline RLBP & 92.88 & 93.44 & 91.83 & 88.58 & 88.48 & 86.04 & 86.31 & 86.19 & 84.31 & 82.31 & 88.04 \\
\hline DRLBP & 93.01 & 93.50 & 91.96 & 88.60 & 87.92 & 85.52 & 84.96 & 85.75 & 83.90 & 81.02 & 87.61 \\
\hline CLBP M & 94.25 & 95.29 & 95.06 & 94.19 & 94.00 & 93.67 & 91.94 & 91.42 & 90.56 & 89.38 & 92.98 \\
\hline CLBP S & 97.50 & 97.52 & 96.75 & 96.23 & 95.85 & 94.65 & 93.85 & 93.56 & 92.77 & 91.65 & 95.03 \\
\hline CPLBP & 97.30 & 96.90 & 96.38 & 96.25 & 95.40 & 94.92 & 94.56 & 93.88 & 93.65 & 92.75 & 95.20 \\
\hline
\end{tabular}

'equal to' and 'less than'. Contrary, LBP can take only two of them 'greater than' and 'less than'. Finally, as can be seen in the table, our proposed descriptor CPLBP achieves a best performance than the other methods in illumination and rotation changes specifically when the radius is high owing to: Firstly, using polar coordinate system that enables to exploit scale and rotation invariance properties for much more robustness approach than other approaches. Secondly, extending the area of neighborhood from one to a region of local pixel neighbors employing radius and angle parameters and represented with the average value increases efficiency in extracting feature without increasing of dimensionality.

Fig. 5 shows the classification accuracy of our approach compared to various state of the art methods using the average of ten different radius $R=\{1,2,3,4$, $5,6,7,8,9,10\}$. It is observed from the fig. that our descriptor achieves best accuracies for all databases. For example, Outex_TC_0010 average accuracy is: $99.86 \%$. 


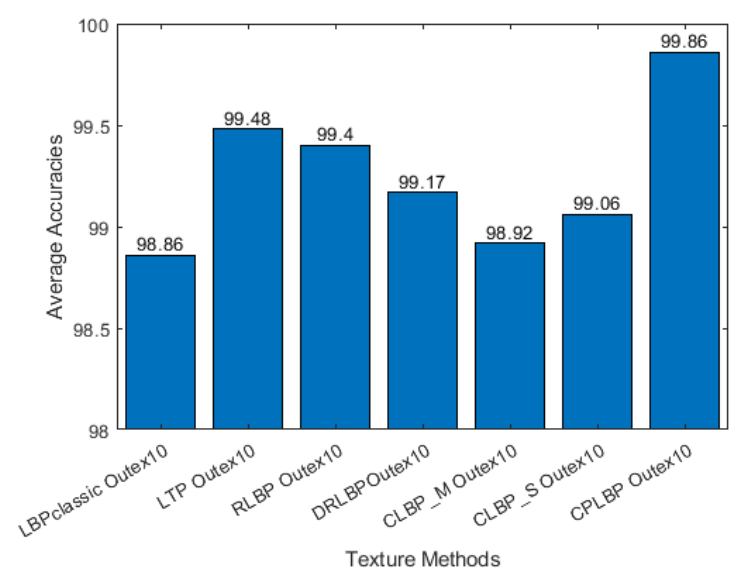

(a)

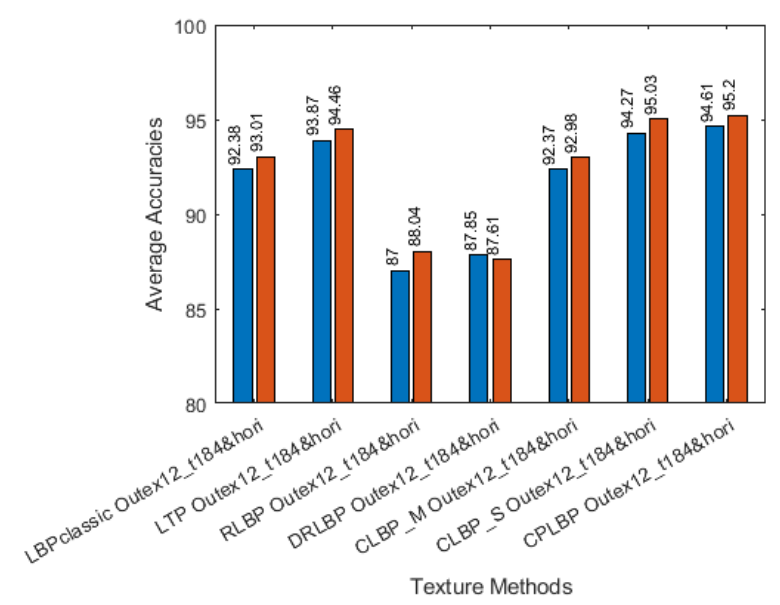

(b)

Fig. 5. The performance of CPLBP (according to the average of different radius) compared to state of the art methods.

\section{EXPERIMENTAL RESULTS 2}

This second experiment aims to evaluate the robustness of our approach using a variety of angles that can decrease or increase the number of parts of local pixel neighbors in the circle. In this study, we adopt the uniform rotation invariant version of LBP to minimize the dimension of feature vector and running time when 12 and 16 neighbors are used. Furthermore, each specific angle of our proposed method is equivalent to an exact number of neighbors on LBP. In this setup, we fixed radius $R=6$ and we varied the angle on five values $\theta=\{\pi / 8, \pi / 6, \pi / 4, \pi / 3, \pi / 2\}$.

Experiment 2-1: Angle evaluate of CPLBP with LBP version on Outex_TC_0010 and 0012

Table 4 shows the classification results on Outex databases 0010 and 0012 (t184 and horizon). As we can seen, the highest classification accuracy of
99.14 is revealed through CPLBP (Outex 10) when $\theta=\pi / 6$. Moreover, for all databases and on each angle value, the best result is obtained by our new descriptors CPLBP which demonstrate the strongest of this method on illumination, rotation and scale changes with the same dimension on the feature pattern of LBP. In general, employing mean value of local regions of pixel neighbors identified by $\theta$ for thresholding instead of one neighbor in LBP is more discriminating, which gives good classification results.

The accuracy of our new proposed approach can be also observed in Fig. 6 after fixing radius as 6 and varying $\theta$ on five angles $\{\pi / 8, \pi / 6, \pi / 4, \pi / 3, \pi / 2\}$. It can be observed that CPLBP preserves high stability and efficiency for various $\theta$ values compared to the original method LBP.

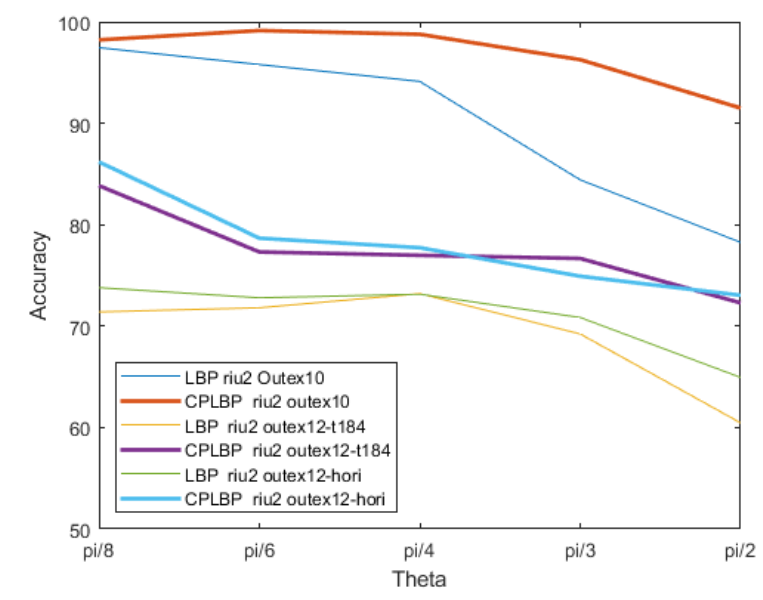

Fig. 6. The performance of CPLBP according to $\theta$ variation using (a) Outex_TC_0010 (b) Outex_TC_0012 t184 and horizon. SOTA

Experiment 2-2: Angle comparative CPLBP with

A comparative study presented in table 5 compares the performance of our new descriptor with state of the art approaches (LTP [Tan and Triggs, 2007], RLBP [Mehta and Egiazarian, 2013], CLBP [Bianconi et al., 2015]) using the most popular challenge databases Outex_TC_0010 and 0012 (t184 and horizon). The significant improvement results of accuracies are marked by our new descriptor CPLBP (riu2) particularly when increasing $\theta$ like $\pi / 3$ and $\pi / 2$ due to the fact that the other descriptors lose a lot of information comes from neighbors and take just a limited number of pixel neighbors which decreases their robustness contrary to CPLBP (riu2) keeping all the details information coming from pixel neighbors.

Fig. 7 plot the results of average classification accuracy as a fonction of $\theta$ values for all databases: $7 \mathrm{a}$ Outex_TC_0010, 7b Outex_TC_0012 t184 and horizon 
Table 4. Classification accuracy (\%) of the CPLBP descriptor for different values of angle $\theta$ with fixed radius $R=6$ on the TC10 and TC12 dataset.

\begin{tabular}{|c|c|c|c|c|c|c|}
\hline \multicolumn{7}{|c|}{ Classification accuracy \% Outex_TC10 and 12} \\
\hline LBP & $\mathrm{N}=16$ & $\mathrm{~N}=12$ & $\mathrm{~N}=8$ & $\mathrm{~N}=6$ & $\mathrm{~N}=4$ & Average \\
\hline CPLBP & $\theta=\pi / 8$ & $\theta=\pi / 6$ & $\theta=\pi / 4$ & $\theta=\pi / 3$ & $\theta=\pi / 2$ & \\
\hline & \multicolumn{6}{|c|}{ Outex_TC10 } \\
\hline LBP riu2 & 97.45 & 95.79 & 94.12 & 84.42 & 78.26 & 90.01 \\
\hline CPLBP riu2 & 98.22 & 99.14 & 98.77 & 96.27 & 91.50 & 96.78 \\
\hline \multicolumn{7}{|c|}{ Outex_TC12 T184 } \\
\hline LBP riu2 & 71.40 & 71.81 & 73.20 & 69.23 & 60.46 & 69.22 \\
\hline CPLBP riu2 & 83.85 & 77.31 & 76.98 & 76.67 & 72.29 & 77.42 \\
\hline \multicolumn{7}{|c|}{ Outex_TC12 horizon } \\
\hline LBP riu2 & 73.79 & 72.79 & 73.15 & 70.85 & 64.94 & 71.11 \\
\hline CPLBP riu2 & 86.19 & 78.67 & 77.73 & 74.92 & 73.04 & 78.11 \\
\hline
\end{tabular}

Table 5. Classification accuracy (\%) of the CPLBP descriptor with the state of the art methods for different values of angle $\theta$ with fixed radius $R=6$ on the TC10 and TC12 dataset.

Classification accuracy \% Outex_TC10 and 12

\begin{tabular}{|c|c|c|c|c|c|c|}
\hline Others descriptors & $\mathrm{N}=16$ & $\mathrm{~N}=12$ & $\mathrm{~N}=8$ & $\mathrm{~N}=6$ & $\mathrm{~N}=4$ & Average \\
\hline CPLBP & $\theta=\pi / 8$ & $\theta=\pi / 6$ & $\theta=\pi / 4$ & $\theta=\pi / 3$ & $\theta=\pi / 2$ & \\
\hline & \multicolumn{6}{|c|}{ Outex_TC10 } \\
\hline LBP riu2 & 97.45 & 95.79 & 94.12 & 84.42 & 78.26 & 90.01 \\
\hline LTP riu2 & 99.51 & 99.44 & 99.14 & 95.72 & 91.81 & 97.12 \\
\hline RLBP riu2 & 97.52 & 95.74 & 94.03 & 89.98 & 78.66 & 91.19 \\
\hline CLBP M riu2 & 97.48 & 97.64 & 94.40 & 85.12 & 70.32 & 88.99 \\
\hline CLBP S riu2 & 97.31 & 95.39 & 93.96 & 83.66 & 78.31 & 89.73 \\
\hline \multirow[t]{2}{*}{ CPLBP riu2 } & 98.22 & 99.14 & 98.77 & 96.27 & 91.50 & 96.78 \\
\hline & \multicolumn{6}{|c|}{ Outex_TC12 T184 } \\
\hline LBP riu2 & 71.40 & 71.81 & 73.20 & 69.23 & 60.46 & 69.22 \\
\hline LTP riu2 & 77.21 & 76.23 & 78.60 & 78.73 & 73.02 & 76.76 \\
\hline RLBP riu2 & 71.54 & 71.92 & 73.19 & 69.02 & 61.83 & 69.50 \\
\hline CLBP $M$ riu2 & 72.23 & 72.13 & 72.13 & 64.54 & 54.50 & 67.11 \\
\hline CLBP S riu2 & 71.67 & 71.25 & 73.02 & 68.90 & 61.58 & 69.28 \\
\hline CPLBP riu2 & 83.85 & 77.31 & 76.98 & 76.67 & 72.29 & 77.42 \\
\hline \multicolumn{7}{|c|}{ Outex_TC12 horizon } \\
\hline LBP riu2 & 73.79 & 72.79 & 73.15 & 70.85 & 64.94 & 71.11 \\
\hline LTP riu2 & 78.67 & 77.00 & 79.79 & 78.81 & 75.50 & 77.95 \\
\hline RLBP riu2 & 73.92 & 72.88 & 73.48 & 70.63 & 65.35 & 71.25 \\
\hline CLBP $M$ riu2 & 72.85 & 73.44 & 72.42 & 64.94 & 54.44 & 67.62 \\
\hline CLBP S riu2 & 73.71 & 73.50 & 73.31 & 70.60 & 64.60 & 71.14 \\
\hline CPLBP riu2 & 86.19 & 78.67 & 77.73 & 74.92 & 73.04 & 78.11 \\
\hline
\end{tabular}

. As illustrated in the fig. 7, CPLBP provides the best results and a high performance even they have small decrease for CPLBP compared to LTP method on Outex_TC_0012. As it maintain before, this diminution due to the thresholding of LTP which takes three relations 'greater than', 'equal to' and 'less than'.

One last remark concerns the running time of our new approach CPLBP. For images with $128 * 128$ pixels like Outex_TC_0010 and under this experimental condition: MATLAB R2018a, Windows system, Intel
Pentium(R) Core(TM) i7-4500U CPU $2.39 \mathrm{GHz}$, and 8 GB memory, the running time consuming with CPLBP is 15 seconds for each image depending on the variation of (Radius, $\theta$ ) which is 10 time bigger than other methods that takes approximately just 0.10 seconds. This time is caused by the computation of average values in each local region which can be seen as a limit of the proposed approach. Although the time consuming of the CPLBP increases, the performance is greatly increased. Specially, when the radius increase because the basic LBP and many other variations are 


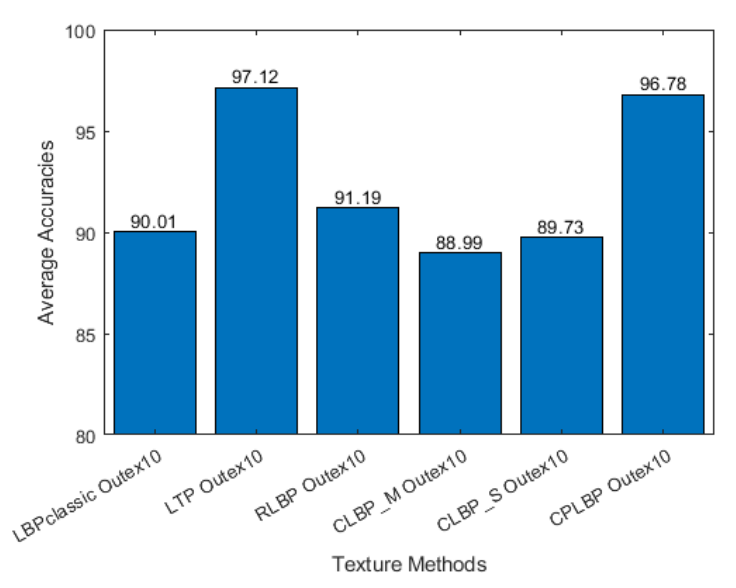

(a)

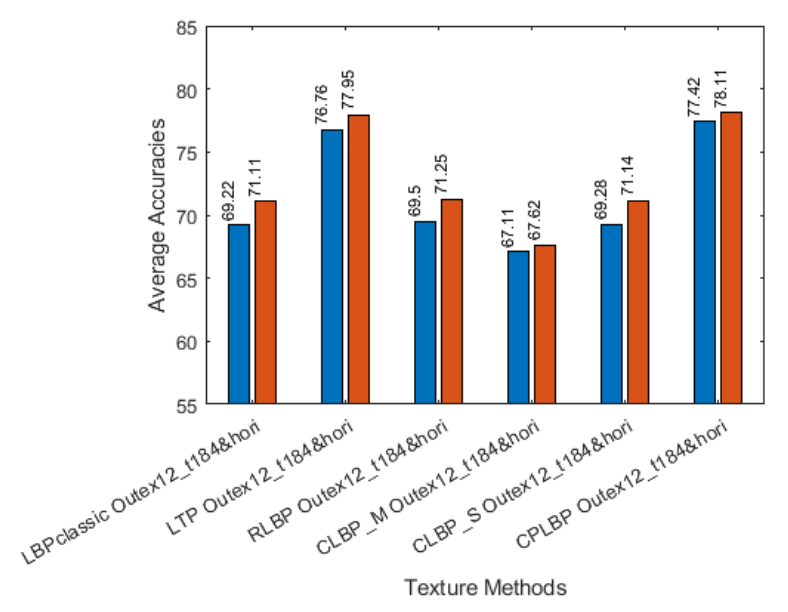

(b)

Fig. 7. The performance of CPLBP according to Average of $\theta$ variation of stat of art methods

used to encode only the local microtexture at the same time they have a problem to capture the macrotexture that is dominant in specific cases. Consequently, our descriptor CPLBP is based on patch mean values, so it is possible to integrate over large areas,therefore the problem of capturing macrotexture is solved.

\section{CONCLUSION}

This paper presented a novel and robust extension of local binary pattern for texture classification. Our approach uses the average of a set of neighbor pixels in the circular angular area instead of one neighbor in the original LBP which allows to get a powerful and discriminating information from images for several cases: fixed $\theta$ and varying $\mathrm{R}$ in the polar coordinates and vice versa. On the Outex databases, the experimental results demonstrate that the suggested approach is more robust than the original one to the changing illumination, scale and rotation. Furthermore, the comparison of our method with the state of art methods shows very good classification accuracies.

\section{REFERENCES}

Ahonen T, Hadid A, Pietikainen M (2006). Face description with local binary patterns: Application to face recognition. IEEE T PATTERN ANAL, 2037-41.

Bianconi F, Gonzalez E, Fernandez, A (2015). Dominant local binary patterns for texture classification: Labelled or unlabelled. PATTERN RECOGN LETT 65, 8-14.
Brahnam S, Jain LC, Nanni L, Lumini A, et al.(2014). Local binary patterns: new variants and applications. Springer.

Chen J, Kellokumpu V, Zhao G, Pietikainen M (2013). Rlbp: Robust local binary pattern., in: BMVC.

Chen JL, Kundu A (1994). Rotation and gray scale transform invariant texture identification using wavelet decomposition and hidden markov model. IEEE T PATTERN ANAL 16, 208-14.

Cui P, Li J, Pan Q, Zhang H (2006). Rotation and scaling invariant texture classification based on radon transform and multiscale analysis. PATTERN RECOGN LETT 27, 408-13.

Deans SR (2007). The Radon transform and some of its applications. Courier Corporation.

Deng H, Clausi DA (2004). Gaussian mrf rotationinvariant features for image classification. IEEE $\mathrm{T}$ PATTERN ANAL, 951-55.

Guo Z, Wang X, Zhou J, You J (2016). Robust texture image representation by scale selective local binary patterns. IEEE T IMAGE PROCESS 25, 687-99.

Guo Z, Zhang L, Zhang D (2010a). A completed modeling of local binary pattern operator for texture classification. IEEE T IMAGE PROCESS $19,1657-63$.

Guo Z, Zhang L, Zhang, D (2010b). Rotation invariant texture classification using lbp variance (lbpv) with global matching. PATTERN RECOGN 43, 706-19.

Kashyap RL, Khotanzad A (1986). A model-based method for rotation invariant texture classification. IEEE T PATTERN ANAL , 472-81.

Liao S, Law MW, Chung AC (2009). Dominant local 
binary patterns for texture classification. IEEE $\mathrm{T}$ IMAGE PROCESS 18, 1107-18.

Liu G, Lin Z, Yu Y (2009). Radon representationbased feature descriptor for texture classification. IEEE T IMAGE PROCESS 18, 921-28.

Liu L, Lao S, Fieguth PW, Guo Y, Wang X, Pietikainen M (2016). Median robust extended local binary pattern for texture classification. IEEE T IMAGE PROCESS 25, 1368-81.

Liu L, Long Y, Fieguth PW, Lao S, Zhao G (2014). Brint: binary rotation invariant and noise tolerant texture classification. IEEE T IMAGE PROCESS 23, 3071-84.

Mehta R, Egiazarian KO (2013). Rotated local binary pattern (rlbp)- rotation invariant texture descriptor, in: ICPRAM.

Nanni L, Lumini A, Brahnam S (2010). Local binary patterns variants as texture descriptors for medical image analysis. ARTIF INTELL MED 49, 117-25.

Nguyen DT, Ogunbona PO, Li W (2013). A novel shape-based nonredundant local binary pattern descriptor for object detection. PATTERN RECOGN 46, 1485-1500.

Ojala T, Pietikainen M, Maenpaa T (2002). Multiresolution gray-scale and rotation invariant texture classification with local binary patterns. IEEE T PATTERN ANAL 24, 971-87.

Pietikainen M, Hadid A, Zhao G, Ahonen T (2011). Computer vision using local binary patterns. volume 40. Springer Science \& Business Media.

Ruiz-del Solar J, Quinteros J (2008). Illumination compensation and normalization in eigenspacebased face recognition: A comparative study of different pre-processing approaches. PATTERN RECOGN LETT 29, 1966-79.

Satpathy A, Jiang X, Eng HL (2014). Lbp-based edgetexture features for object recognition. IEEE $T$ IMAGE PROCESS 23, 1953-64.

Tabatabaei SM, Chalechale A (2019). Noise-tolerant texture feature extraction through directional thresholded local binary pattern. VISUAL COMPUT , 1-21.

Tan X, Triggs B (2007). Enhanced local texture feature sets for face recognition under difficult lighting conditions, in: International workshop on analysis and modeling of faces and gestures, Springer. pp. 168-82.

Trefny J, Matas J (2010). Extended set of local binary patterns for rapid object detection, in: LECT NOTES COMPUT SC, pp. 1-7.

Tuceryan M, Jain AK (1993). Texture analysis, in:
Handbook of pattern recognition and computer vision. WORLD SCI, pp. 235-76.

Zhao G, Pietikainen M (2007). Dynamic texture recognition using local binary patterns with an application to facial expressions. IEEE $\mathrm{T}$ PATTERN ANAL 29, 915-28. 